\title{
Analytic algorithms for Some Models of Nonlinear Age-Structured Population Dynamics and Epidemiology
}

\author{
Vipul K. Baranwal, Ram K. Pandey, Manoj P. Tripathi, Om. P. Singh \\ Department of Applied Mathematics, Institute of Technology, Banaras Hindu University, \\ Varanasi, India \\ E-mail:singhom@gmail.com,opsingh.apm@itbhu.ac.in \\ Received January 4, 2011; revised February 7, 2011; accepted February 9, 2011
}

\begin{abstract}
Three analytic algorithms based on Adomian decomposition, homotopy perturbation and homotopy analysis methods are proposed to solve some models of nonlinear age-structured population dynamics and epidemiology. Truncating the resulting convergent infinite series, we obtain numerical solutions of high accuracy for these models. Three numerical examples are given to illustrate the simplicity and accuracy of the methods.
\end{abstract}

Keywords: Age-Structured Population Models, Population Dynamics, SIS Epidemic Models, Adomian Decomposition Method, Homotopy Perturbation and Homotopy Analysis Methods.

\section{Introduction}

Individuals in a structured population are distinguished by age, size, maturity and some other individual physical characteristics. The basic assumption when modelling the evaluation of such a population is that the structure of the population with respect to these individual physical characteristics at a given time, and possibly some environmental inputs as time evolves, completely determines the dynamical behaviours of the population. Mathematical models describing this evolution have attracted a considerable amount of interest among scientists as a tool for modelling the interaction of different population communities in such diverse fields as demography, epidemiology, ecology, cell kinetics, tumer growth etc.

For a long time, there has been an interest in modelling population dynamics. The first discrete population model appears in Liber Abaci by Leonardo Pisano in 1228 [1], which gives rise to the celebrated Fibonacci sequences. The simplest continuous model is due to Malthus in 1798 [2]. His model is an unstructured one and it leads to an exponential growth of the population which is usually invalid for large time. Forty years later, in 1838, Verhulst proposed a logistic model which impose a maximum size for the population by considering the effects of crowding and the limitation of resources.

In order to build adequate models for population dynamics, some detail concerning individual behaviour and its effects on vital rates of growth, production, and death must be included. Perhaps the most natural way to consider such effects is to introduce the age variable into the model describing the population dynamics.

Among the first continuous population models incorporating age effects were those of Sharpe and Lotka [3] and McKendrick [4]. Basically, the Sharpe-LotkaMcKendrick models assume that birth and mortality processes are linear functions of population density. In 1974, Gurtin and MacCamy [5] proposed a nonlinear age structured model. The allowed the mortality rate and the fertility rate to be affected by the total population, which is true for the most real cases. Their model generalizes Verhulst's, and under reasonable assumptions on the vital rates of the population, results in a logistic model with bounded growth.

We consider the following nonlinear age-structured population model [5] and name it as model (A):

$$
\begin{gathered}
\frac{\partial u}{\partial t}+\frac{\partial u}{\partial x}+\mu(x, P(t)) u=0,0 \leq x<A, t>0, \\
u(x, 0)=u_{0}(x), 0 \leq x<A, \\
u(0, t)=\int_{0}^{A} \beta(x, P(t)) u(x, t) \mathrm{d} x, t>0, \\
P(t)=\int_{0}^{A} u(x, t) \mathrm{d} x, t \geq 0,
\end{gathered}
$$

and some related epidemic models introduced latter, where $u(x, t)$ is the population density (or age-density) 
with respect to age $x$ of a population at time $t$. The units of $u(x, t)$ are given in units of population divided by units of time. Hence, the total number of individuals between ages $a$ and $a+\Delta a$ is given by $\int_{a}^{a+\Delta a} u(x, t) \mathrm{d} x$, where $u(x, t)$ is taken to be smooth function of $(x, t)$. The model (A) describes the evolution of the age-density $u(x, t)$ of a population, with maximum age $A<\infty$, whose growth is regulated by the vital rates $\beta$ and $\mu$ [5-7]. To be specific, $\beta$ and $\mu$ denote fertility and death rate respectively. If $\mu=\mathrm{d}_{1}(x)+\mathrm{d}_{2}(x) P(t)$ and $\beta=b_{1}(x)-b_{2}(x) P(t)$, then the model (A) reduces to what we call model (B):

$$
\begin{gathered}
\frac{\partial u}{\partial t}+\frac{\partial u}{\partial x}=-\left[\mathrm{d}_{1}(x)+\mathrm{d}_{2}(x) P(t)\right] u(x, t), \\
0 \leq x<A, t \geq 0, \\
u(x, 0)=u_{0}(x), 0 \leq x<A, \\
u(0, t)=\int_{0}^{A}\left[b_{1}(\zeta)-b_{2}(\zeta) P(t)\right] u(\zeta, t) \mathrm{d} \zeta, t \geq 0, \\
P(t)=\int_{0}^{A} u(x, t) \mathrm{d} x, t \geq 0,
\end{gathered}
$$

where $\mathrm{d}_{1}(x)$ is the natural death rate (without considering competition), $\mathrm{d}_{2}(x) P(t)$ is the increase of death rate due to competition, $b_{1}(x)$ is the natural fertility rate (without considering competition), $b_{2}(x) P(t)$ is the decrease of fertility rate considering competition. Equation (1.3) or (1.7), also called the renewal equation, gives the number of new born individuals introduced into the population. The value of $u(0, t)$ at any time $t$ depends on the age distribution of the population at that time.

The nonlocal boundary condition (1.7) complicates the application of standard numerical techniques such as finite difference, finite elements, spectral methods and so on [8]. So, to avoid the complexity involved in applying those numerical methods to the population model, it is important to convert the nonlocal boundary value problem into more desirable forms. However, it turns out to be a hard work in many cases. Therefore, for nonlinear age-structured population models, there are only few methods for solving them. In recent years, the numerical approximation of the model (A) has been studied by several authors like Abia and Lopez-Marcos, they applied difference schemes based on Runge-Kutta method and other numerical integration techniques to solve it [9-11]. In [12], Kim and Park developed an upwind scheme for the model (A). Iannelli et al. [13] solved it by using splitting methods. Reproducing kernel method was successfully applied by Cui and Chen [14] and Krzyzanowski et al. gave a discontinuous Galerkin method for non linear age structured population model [15]. Norhayati and Wake [16] used Laplace transform technique to solve and analysed the existence of steady age distribution and its stability.

Recently methods like Adomian decomposition method (ADM), homotopy perturbation method (HPM), homotopy analysis method (HAM) have been used successfully to solve a variety of non linear problems [17-20]. Dehghan and Salehi [21] used VIM and ADM to solve the delay logistic equation which has been extensively used as models in biology with particular emphasis on population dynamics. In 2009, Li [8] applied VIM to solve the model (B) with great success, but ADM, HPM and HAM have yet not been used for the purpose.

The aim of the present paper is to apply these techniques for the numerical evaluation of the non linear age-structured population model (B). The basic ideas of these methods apply to other problems related to (B). In fact the same approaches are used for approximation of the age-structured SIS model.

The paper is organized as follows. In sections 2-4, we introduce the algorithms based on ADM, HPM and HAM respectively. In sec. 5, we apply these algorithms on some numerical experiments and finally conclusions are given in sec. 6 .

\section{Adomian Decomposition Method (ADM)}

In this section we give a brief outline of ADM for solving nonlinear age-structured population model (NASPM). Equation (1.5) may be written in the operator form as

$$
\begin{gathered}
L_{t}(u(x, t))+L_{x}(u(x, t))+\mathrm{d}_{1}(x) u(x, t) \\
+\mathrm{d}_{2}(x) N(u(x, t))=0,
\end{gathered}
$$

where the notations $L_{t}=\frac{\partial}{\partial t}$ and $L_{x}=\frac{\partial}{\partial x}$ symbolize the linear differential operators and the notation $N(u(x, t))=u(x, t) \int_{0}^{A} u(x, t) \mathrm{d} x$ symbolizes the nonlinear operator.

The inverse operator $L_{t}^{-1}$, is defined by $L_{t}^{-1}(\cdot)=\int_{0}^{t}(\cdot) \mathrm{d} \xi$.

Thus, applying the inverse operator $L_{t}^{-1}$ to Equation (2.1), we get

$$
\begin{aligned}
u(x, t)= & u(x, 0)-L_{t}^{-1}\left[L_{x}(u(x, t))\right. \\
& \left.+\mathrm{d}_{1}(x) u(x, t)+\mathrm{d}_{2}(x) N(u(x, t))\right] .
\end{aligned}
$$

The ADM [17,18] assumes an infinite series solution for the unknown functions $u(x, t)$, given by 


$$
u(x, t)=\sum_{n=0}^{\infty} u_{n}(x, t) .
$$

The nonlinear operator $N(u(x, t))$ is decomposed as

$$
N(u(x, t))=\sum_{n=0}^{\infty} A_{n}\left(u_{0}, u_{1}, u_{2}, \cdots, u_{n}\right),
$$

where $A_{n}$ is an approximate Adomian's polynomial which can be calculated for all forms of nonlinearity according to specific algorithms constructed by Adomian

$$
A_{0}=N\left(u_{0}\right), A_{1}=N^{\prime}\left(u_{0}\right) u_{1}, A_{2}=N^{\prime}\left(u_{0}\right) u_{2}+N^{\prime \prime}\left(u_{0}\right) \frac{u_{1}^{2}}{2 !}, A_{3}=N^{\prime}\left(u_{0}\right) u_{3}+N^{\prime \prime}\left(u_{0}\right) u_{1} u_{2}+N^{\prime \prime \prime}\left(u_{0}\right) \frac{u_{1}^{3}}{3 !}, \cdots .
$$

Substituting (2.3) and (2.4) in (2.2), we get

$$
\left.\sum_{n=0}^{\infty} u_{n}=u(x, 0)-L_{t}^{-1}\left[L_{x} \sum_{n=0}^{\infty} u_{n}+\mathrm{d}_{1}(x) \sum_{n=0}^{\infty} u_{n}+\mathrm{d}_{2}(x) \sum_{n=0}^{\infty} A_{n}\right)\right] .
$$

Identifying the zeroth component $u_{0}(x, t)$ by the initial condition $u(x, 0)$, we obtain the subsequent com-

$$
\left.u_{0}(x, t)=u(x, 0), \quad u_{n+1}(x, t)=-L_{t}^{-1}\left[L_{x}\left(u_{n}(x, t)\right)+\mathrm{d}_{1}(x) u_{n}(x, t)+\mathrm{d}_{2}(x) A_{n}\right)\right] .
$$

\section{Homotopy Perturbation Method (HPM)}

In this method, using the homotopy technique of topology, a homotopy is constructed with an embedding parameter $p \in[0,1]$ which is considered as a "small parameter". This method became very popular amongst the scientists and engineers, even though it involves continuous deformation of a simple problem into a more difficult problem under consideration. Most of the perturbation methods depend on the existence of a small perturbation parameter but many nonlinear problems have no small perturbation parameter at all. Many new methods have been proposed in the late nineties to solve such nonlinear equation devoid of such small parameters. Late 1990s saw a surge in applications of homotopy theory in the scientific and engineering computations [19]. When the homotopy theory is coupled with perturbation theory it provides a powerful mathematical tool. To illustrate the basic concept of HPM, consider the following nonlinear functional equation

$$
A(u)=f(r), r \in \Omega,
$$

with the boundary conditions $B\left(u, \frac{\partial u}{\partial n}\right)=0, r \in \partial \Omega$,

where $A$ is a general functional operator, $B$ is a boundary operator, $f(r)$ is a known analytic function, and $\partial \Omega$ is the boundary of the domain $\Omega$. The operator $A$ is decomposed as $A=L+N$, where $L$ is the linear and $N$ is the nonlinear operator. Hence Equation (3.1) can be written as

$$
L(u)+N(u)-f(r)=0, r \in \Omega .
$$

We construct a homotopy $v(r, p): \Omega \times[0,1] \rightarrow R$ satisfying

$$
\begin{gathered}
H(v, p)=(1-p)\left[L(v)-L\left(u_{0}\right)\right]+p[A(v)-f(r)]=0, \\
p \in[0,1], r \in \Omega .
\end{gathered}
$$

Hence,

$$
H(v, p)=L(v)-L\left(u_{0}\right)+p L\left(u_{0}\right)+p[N(v)-f(r)]=0,
$$

where $u_{0}$ is an initial approximation for the solution of (3.1). As

$$
H(v, 0)=L(v)-L\left(u_{0}\right) \text { and } H(v, 1)=A(v)-f(r) \text {, }
$$

it shows that $H(v, p)$ continuously traces an implicitly defined curve from a starting point $H\left(u_{0}, 0\right)$ to a solution $H(v, 1)$. The embedding parameter $p$ increases monotonously from zero to one as the trivial linear part $L(u)=0$ deforms continuously to the original problem $A(u)=f(r)$. The embedding parameter $p \in[0,1]$ can be considered as an expanding parameter [19] to obtain

$$
v=v_{0}+p v_{1}+p^{2} v_{2}+\cdots .
$$

The solution is obtained by taking the limit as $p$ tends to 1 in equation.(3.5). Hence

$$
u=\lim _{p \rightarrow 1} v=v_{0}+v_{1}+v_{2}+\cdots
$$

The series (3.6) converges for most cases and the rate of convergence depends on $A(u)-f(r)$.

For nonlinear age-structured population model, we choose the initial approximation $u_{0}(x, t)=u_{0}(x)$, and construct the following homotopy: 


$$
(1-p)\left(\frac{\partial v(x, t)}{\partial t}-\frac{\partial u_{0}(x, t)}{\partial t}\right)+p\left(\frac{\partial v(x, t)}{\partial t}+\frac{\partial v(x, t)}{\partial x}+\left(\mathrm{d}_{1}(x)+\mathrm{d}_{2}(x) \int_{0}^{A} v(x, t) \mathrm{d} x\right) v(x, t)\right)=0,
$$

which is equivalent to

$$
\frac{\partial v(x, t)}{\partial t}-\frac{\partial u_{0}(x, t)}{\partial t}+p \frac{\partial u_{0}(x, t)}{\partial t}+p\left(\frac{\partial v(x, t)}{\partial x}+\left(\mathrm{d}_{1}(x)+\mathrm{d}_{2}(x) \int_{0}^{A} v(x, t) \mathrm{d} x\right) v(x, t)\right)=0,
$$

where $p \in[0,1]$ is an embedding parameter. Using the parameter $p$, we expand the solution in the following form

$$
v(x, t)=v_{0}(x, t)+p v_{1}(x, t)+p^{2} v_{2}(x, t)+p^{3} v_{3}(x, t)+\cdots
$$

Substituting Equation (3.9) into Equation (3.8), and

equating the terms with the identical powers of $p$, we obtain:

$$
\begin{aligned}
& p^{0}: \frac{\partial v(x, t)}{\partial t}-\frac{\partial u_{0}(x, t)}{\partial t}=0, v_{0}(x, 0)=u(x, 0), \\
& p^{1}: \frac{\partial u_{1}(x, t)}{\partial t}+\frac{\partial u_{0}(x, t)}{\partial t}+\frac{\partial v_{0}(x, t)}{\partial x}+\mathrm{d}_{1}(x) v_{0}(x, t)+\mathrm{d}_{2}(x) v_{0}(x, t) \int_{0}^{A} v_{0}(x, t) \mathrm{d} x=0, v_{1}(x, 0)=0, \\
& p^{2}: \frac{\partial v_{2}(x, t)}{\partial t}+\frac{\partial v_{1}(x, t)}{\partial x}+\mathrm{d}_{1}(x) v_{1}(x, t)+\mathrm{d}_{2}(x) v_{1}(x, t) \int_{0}^{A} v_{0}(x, t) \mathrm{d} x+\mathrm{d}_{2}(x) v_{0}(x, t) \int_{0}^{A} v_{1}(x, t) \mathrm{d} x=0, \\
& \quad v_{2}(x, 0)=0, \\
& p^{3}: \frac{\partial v_{3}(x, t)}{\partial t}+\frac{\partial v_{2}(x, t)}{\partial x}+\mathrm{d}_{1}(x) v_{2}(x, t)+\mathrm{d}_{2}(x) v_{2}(x, t) \int_{0}^{A} v_{0}(x, t) \mathrm{d} x+\mathrm{d}_{2}(x) v_{1}(x, t) \int_{0}^{A} v_{1}(x, t) \mathrm{d} x \\
& \quad+\mathrm{d}_{2}(x) v_{0}(x, t) \int_{0}^{A} v_{2}(x, t) \mathrm{d} x=0, \quad v_{3}(x, 0)=0, \cdots
\end{aligned}
$$

We use the iterative scheme (3.10) to compute the various $v_{i}$,s. Hence the solution of Equation (1.5) is given by,

$$
u(x, t)=\lim _{p \rightarrow 1} v(x, t)=\sum_{m=0}^{\infty} v_{m}(x, t) .
$$

\section{Homotopy Analysis Method (HAM)}

Homotopy analysis method (HAM) was first proposed by Liao [20] based on homotopy, a fundamental concept in topology and differential geometry. The HAM is based on construction of homotopy which continuously deforms an initial guess approximation to the exact solution of the given problem. An auxiliary linear operator is chosen to construct the homotopy and an auxiliary linear parameter is used to control the region of convergence of the solution series, which is not possible in the other methods like perturbation techniques, homotopy perturbation methods, decomposition methods. The HAM provides the greater flexibility in choosing initial approximations and auxiliary linear operators and hence a complicated nonlinear problem can be transformed into infinite number simpler, linear sub problems as shown by Liao and Tan [22].

Here we give a brief description of HAM [20] to han- dle the general non linear problem,

$$
N[u(x, t)]=0, t>0,
$$

where $N$ is a nonlinear operator and $u(x, t)$ is unknown function of the independent variables $x, t$. Liao [20] constructed the zero order deformation equation

$$
\begin{gathered}
(1-q) L\left[\varphi(x, t ; q)-u_{0}(x, t)\right] \\
=q \hbar H(x, t) N[\varphi(x, t ; q)],
\end{gathered}
$$

where $q \in[0,1]$ is the homotopy or embedding parameter, $\hbar \neq 0$ is an auxiliary parameter, $H(x, t) \neq 0$ an auxiliary function, $L$ is an auxiliary linear operator, $u_{0}(x, t)$ an initial guess of $u(x, t)$ and $\varphi(x, t ; q)$ is an unknown function.

Putting $q=0$, and $q=1$, in Equation (4.2), we see that

$$
\begin{aligned}
& \varphi(x, t ; 0)=u_{0}(x, t), \\
& \varphi(x, t ; 1)=u(x, t),
\end{aligned}
$$

Therefore, according to Equations (4.3) \& (4.4), $\varphi(x, t ; q)$ deforms continuously from the initial guess $u_{0}(x, t)$ to the exact solution $u(x, t)$ as the embedding parameter $q$ increases from 0 to 1 . Liao [20] expanded $\varphi(x, t ; q)$ in a Taylor series as follows 


$$
\varphi(x, t ; q)=u_{0}(x, t)+\sum_{m=1}^{\infty} u_{m}(x, t) q^{m},
$$

where

$$
u_{m}(x, t)=\left.\frac{1}{m !} \frac{\partial^{m} \varphi(x, t ; q)}{\partial q^{m}}\right|_{q=0} .
$$

The convergence of the series (4.5) is controlled by $\hbar$. Assume that the auxiliary parameter $\hbar$, the auxiliary function $H$, the initial approximation $u_{0}(x, t)$, and the auxiliary linear operator $L$ are so properly chosen that the series (4.5) converges at $q=1$. Then, at $q=1$ and using (4.4) the series (4.5) gives the exact solution $u(x, t)$ as

$$
u(x, t)=u_{0}(x, t)+\sum_{m=1}^{\infty} u_{m}(x, t)
$$

The above expression provides us with a relationship between the initial guess $u_{0}(x, t)$ and the exact solution $u(x, t)$ by means of the terms $u_{m}(x, t)(m=1,2,3, \cdots)$, which are still to be determined. The process of their evaluations is given as follows :

Differentiating the zero order deformation Equation (4.2) $m$ times with respect to embedding parameter $q$, then setting $q=0$ and dividing by $m$ !, we get the following $m t h$-order deformation equation,

$$
L\left[u_{m}(x, t)-\chi_{m} u_{m-1}(x, t)\right]=\hbar H(t) R_{m}\left(\boldsymbol{u}_{m-1}(x, t)\right),
$$

where

$$
\begin{gathered}
R_{m}\left(\boldsymbol{u}_{m-1}\right)=\left.\frac{1}{(m-1) !} \frac{\partial^{m-1} N[\varphi(x, t ; q)]}{\partial q^{m-1}}\right|_{q=0}, \\
\boldsymbol{u}_{m}=\left\{u_{0}(x, t), u_{1}(x, t), u_{2}(x, t) \cdots u_{m}(x, t)\right\}, \\
\chi_{m}=\left\{\begin{array}{l}
0, m \leq 1 \\
1, \text { otherwise }
\end{array} .\right.
\end{gathered}
$$

For any given operators $L$ and $N$ we get the $m$ th order deformation Equation (4.8) and solving it we get different $u_{m}(x, t)$. The solution of problem (4.1) is obtained by putting these $u_{m}(x, t)$ 's in (4.7) and choosing a suitable value of $\hbar$ for the convergence of the series. The symbolic computation software like Maple and Mathematica can solve (4.8) easily.

\section{Numerical Applications}

In this paper, we apply ADM, HPM and HAM to solve the nonlinear age-structured population models. In the following examples $\tilde{u}_{n}(x, t)$ will denote an approximate solution of the problem under consideration, obtained by truncating the solution series (4.7) at level $m=n$. Also $E_{n}(\hbar)=\left|u_{\text {exact }}(x, t)-\tilde{u}_{n}(x, t)\right|$ denotes the error between exact and approximate solution at $\hbar$. In Table 1, $E_{1}$ denote the error between exact solution and approximate solution obtained by reproducing kernel method [14].

Example 5.1 Consider the following nonlinear agestructured population model [8, 14].

$$
\begin{gathered}
\frac{\partial u(x, t)}{\partial t}+\frac{\partial u(x, t)}{\partial x}=-P(t) u(x, t), t \geq 0,0 \leq x<A, \\
u(x, 0)=\frac{\mathrm{e}^{-x}}{2}, 0 \leq x<A \\
u(0, t)=P(t), t \geq 0 \\
P(t)=\int_{0}^{A} u(x, t) \mathrm{d} x, t \geq 0
\end{gathered}
$$

where, $A=+\infty$, with $u(x, t)=\frac{\mathrm{e}^{-x}}{1+\mathrm{e}^{-t}}, t \geq 0, x \geq 0$, as the exact solution of (5.1).

\section{Case (a) Solution by ADM}

Rewriting Equation (5.1) in the operator form

$$
L_{t} u(x, t)=-L_{x} u(x, t)-u(x, t) \int_{0}^{A} u(x, t) \mathrm{d} x,
$$

taking the initial approximation $u_{0}(x, t)=\frac{\mathrm{e}^{-x}}{2}$, and using the recursive formula (2.7), we find that all the even iterates $u_{2 n}=0, n=0,1,2,3, \cdots$ and

$$
\begin{gathered}
u_{1}(x, t)=t \frac{\mathrm{e}^{-x}}{4}, u_{3}(x, t)=-t^{3} \frac{\mathrm{e}^{-x}}{48}, \\
u_{5}(x, t)=t^{5} \frac{\mathrm{e}^{-x}}{480}, u_{7}(x, t)=-t^{7} \frac{\mathrm{e}^{-x}}{80640}, \cdots .
\end{gathered}
$$

Hence, the solution is given by

$$
\begin{aligned}
u(x, t) & =\lim _{N \rightarrow \infty} \sum_{n=0}^{N} u_{n}(x, t) \\
& =\mathrm{e}^{-x}\left(\frac{1}{2}+\frac{t}{4}-\frac{t^{3}}{48}+\frac{t^{5}}{480}-\frac{t^{7}}{80640}+\cdots\right) \\
& =\frac{\mathrm{e}^{-x}}{1+\mathrm{e}^{-t}}
\end{aligned}
$$

which is the exact solution.

\section{Case (b) Solution by HPM}

Taking the same initial approximation $u_{0}(x, t)=\frac{\mathrm{e}^{-x}}{2}$, and using the equations (3.10), we find that all the even iterates $v_{2 n}=0, n=0,1,2,3, \cdots$ and 
Table 1. Comparison between HAM and reproducing kernel method solutions.

\begin{tabular}{|c|c|c|c|c|c|}
\hline \multicolumn{2}{|c|}{ Nodes } & \multirow{2}{*}{$\begin{array}{c}\text { Exact Solution } \\
u(x, t)\end{array}$} & \multirow{2}{*}{$\begin{array}{c}\text { Approximate Solution } \\
\tilde{u}_{7}(x, t), \hbar=-1\end{array}$} & \multirow{2}{*}{$\begin{array}{c}\text { Error } \\
E_{7}(-1)=\left|u(x, t)-\tilde{u}_{7}(x, t)\right|\end{array}$} & \multirow{2}{*}{$\begin{array}{c}\text { Error [14] } \\
E_{1}\end{array}$} \\
\hline $\mathrm{t}$ & $\mathrm{X}$ & & & & \\
\hline 0.00 & 0.00 & 0.5 & 0.5 & 0 & 0 \\
\hline 0.20 & 0.00 & 0.549834 & 0.549834 & $1.08906 \mathrm{E}-11$ & $2.6162 E-02$ \\
\hline 0.40 & 0.00 & 0.598688 & 0.598688 & $5.50928 E-09$ & $4.4321 \mathrm{E}-02$ \\
\hline 0.60 & 0.00 & 0.645656 & 0.645656 & $2.07654 \mathrm{E}-07$ & $4.4703 \mathrm{E}-02$ \\
\hline 0.80 & 0.00 & 0.689974 & 0.689972 & $2.69192 \mathrm{E}-06$ & $3.6536 \mathrm{E}-02$ \\
\hline 1.00 & 0.00 & 0.731059 & 0.731039 & $1.93921 \mathrm{E}-05$ & $1.1234 \mathrm{E}-02$ \\
\hline 0.00 & 1.00 & 0.18394 & 0.18394 & 0 & 0 \\
\hline 0.20 & 1.00 & 0.202273 & 0.202273 & $4.00643 E-12$ & $6.467 E-03$ \\
\hline 0.40 & 1.00 & 0.220245 & 0.220245 & $2.02675 E-09$ & $1.2146 \mathrm{E}-02$ \\
\hline 0.60 & 1.00 & 0.237524 & 0.237524 & $7.63918 E-08$ & $1.4046 \mathrm{E}-02$ \\
\hline 0.80 & 1.00 & 0.253827 & 0.253826 & $9.90302 E-07$ & $7.887 \mathrm{E}-03$ \\
\hline 1.00 & 1.00 & 0.268941 & 0.268934 & $7.13396 \mathrm{E}-06$ & $-5.552 E-03$ \\
\hline 0.00 & 2.00 & 0.0676676 & 0.0676676 & 0 & 0 \\
\hline 0.20 & 2.00 & 0.0744119 & 0.0744119 & $1.47389 \mathrm{E}-12$ & $5.5869 \mathrm{E}-03$ \\
\hline 0.40 & 2.00 & 0.0810236 & 0.0810236 & $7.456 \mathrm{E}-10$ & $9.6181 \mathrm{E}-03$ \\
\hline 0.60 & 2.00 & 0.0873801 & 0.0873801 & $2.8103 E-08$ & $1.03579 \mathrm{E}-02$ \\
\hline 0.80 & 2.00 & 0.0933779 & 0.0933775 & $3.64312 E-07$ & $5.4159 \mathrm{E}-03$ \\
\hline 1.00 & 2.00 & 0.098938 & 0.0989354 & $2.62444 \mathrm{E}-06$ & $6.5159 \mathrm{E}-03$ \\
\hline 0.00 & 3.00 & 0.0248935 & 0.0248935 & 0 & 0 \\
\hline 0.20 & 3.00 & 0.0273746 & 0.0273746 & $5.42216 \mathrm{E}-13$ & $4.4722 \mathrm{E}-03$ \\
\hline 0.40 & 3.00 & 0.0298069 & 0.0298069 & $2.74291 \mathrm{E}-10$ & $7.3509 E-03$ \\
\hline 0.60 & 3.00 & 0.0321453 & 0.0321453 & $1.03385 E-08$ & $8.2104 \mathrm{E}-03$ \\
\hline 0.80 & 3.00 & 0.0343518 & 0.0343517 & $1.34023 E-07$ & $6.4072 E-03$ \\
\hline 1.00 & 3.00 & 0.0363973 & 0.0363963 & $9.65477 \mathrm{E}-07$ & $2.9965 E-03$ \\
\hline 0.00 & 4.00 & 0.00915782 & 0.00915782 & 0 & 0 \\
\hline 0.20 & 4.00 & 0.0100706 & 0.0100706 & $1.99471 \mathrm{E}-13$ & $1.8242 \mathrm{E}-03$ \\
\hline 0.40 & 4.00 & 0.0109653 & 0.0109653 & $1.00906 \mathrm{E}-10$ & $3.2864 \mathrm{E}-03$ \\
\hline 0.60 & 4.00 & 0.0118256 & 0.0118256 & $3.80332 \mathrm{E}-09$ & $3.7749 \mathrm{E}-03$ \\
\hline 0.80 & 4.00 & 0.0126373 & 0.0126373 & $4.93043 E-08$ & $3.1609 E-03$ \\
\hline 1.00 & 4.00 & 0.0133898 & 0.0133894 & $3.55179 \mathrm{E}-07$ & $1.8526 \mathrm{E}-03$ \\
\hline 0.00 & 5.00 & 0.00336897 & 0.00336897 & 0 & 0 \\
\hline 0.20 & 5.00 & 0.00370475 & 0.00370475 & $7.33805 E-14$ & $-3.3172 E-04$ \\
\hline 0.40 & 5.00 & 0.00403393 & 0.00403393 & $3.71212 E-11$ & $-2.5414 \mathrm{E}-04$ \\
\hline 0.60 & 5.00 & 0.0043504 & 0.0043504 & $1.39916 \mathrm{E}-09$ & $-1.3464 \mathrm{E}-04$ \\
\hline 0.80 & 5.00 & 0.00464901 & 0.00464899 & $1.8138 \mathrm{E}-08$ & $-3.5880 \mathrm{E}-04$ \\
\hline 1.00 & 5.00 & 0.00492583 & 0.0049257 & $1.30663 \mathrm{E}-07$ & $-8.72 E-04$ \\
\hline 0.00 & 6.00 & 0.00123938 & 0.00123938 & 0 & 0 \\
\hline 0.20 & 6.00 & 0.0013629 & 0.0013629 & $2.69953 E-14$ & $8.9154 \mathrm{E}-04$ \\
\hline 0.40 & 6.00 & 0.001484 & 0.001484 & $1.36561 \mathrm{E}-11$ & $2.3233 E-03$ \\
\hline 0.60 & 6.00 & 0.00160042 & 0.00160042 & $5.14724 \mathrm{E}-10$ & $3.85194 \mathrm{E}-03$ \\
\hline 0.80 & 6.00 & 0.00171028 & 0.00171027 & $6.67261 \mathrm{E}-09$ & $5.38069 \mathrm{E}-03$ \\
\hline 1.00 & 6.00 & 0.00181211 & 0.00181206 & $4.80683 \mathrm{E}-08$ & $6.49366 \mathrm{E}-03$ \\
\hline 0.00 & 8.00 & 0.000167731 & 0.000167731 & 0 & 0 \\
\hline 0.20 & 8.00 & 0.000184449 & 0.000184449 & $3.65338 E-15$ & $4.58849 E-03$ \\
\hline 0.40 & 8.00 & 0.000200837 & 0.000200837 & $1.84816 \mathrm{E}-12$ & $6.31876 \mathrm{E}-03$ \\
\hline 0.60 & 8.00 & 0.000216594 & 0.000216593 & $6.96603 E-11$ & $9.81151 \mathrm{E}-03$ \\
\hline 0.80 & 8.00 & 0.000231461 & 0.00023146 & $9.03039 E-10$ & $1.30453 \mathrm{E}-02$ \\
\hline 1.00 & 8.00 & 0.000245243 & 0.000245236 & $6.50533 E-09$ & $1.6041 \mathrm{E}-02$ \\
\hline 0.00 & 10.00 & 0.0000227 & 0.0000227 & 0 & 0 \\
\hline 0.20 & 10.00 & 0.0000249624 & 0.0000249624 & $4.94433 E-16$ & $2.14306 \mathrm{E}-04$ \\
\hline 0.40 & 10.00 & 0.0000271804 & 0.0000271804 & $2.50121 E-13$ & $1.14205 E-03$ \\
\hline 0.60 & 10.00 & 0.0000293128 & 0.0000293127 & $9.42749 \mathrm{E}-12$ & $2.52654 \mathrm{E}-03$ \\
\hline 0.80 & 10.00 & 0.0000313248 & 0.0000313247 & 1.22213E - 10 & $3.89648 E-03$ \\
\hline 1.00 & 10.00 & 0.00003319 & 0.0000331891 & $8.80401 \mathrm{E}-10$ & $5.20734 \mathrm{E}-03$ \\
\hline
\end{tabular}




$$
\begin{aligned}
& v_{1}(x, t)=t \frac{\mathrm{e}^{-x}}{4}, v_{3}(x, t)=-t^{3} \frac{\mathrm{e}^{-x}}{48} \\
& v_{5}(x, t)=t^{5} \frac{\mathrm{e}^{-x}}{480}, v_{7}(x, t)=-t^{7} \frac{\mathrm{e}^{-x}}{80640}, \cdots
\end{aligned}
$$

Thus we see that the various terms obtained by using HPM are same as those obtained by using ADM. In general, the ADM solution is a part of HPM solution.

Substituting these values in Equation (3.11), the solution is given by

$$
\begin{aligned}
u(x, t) & =\lim _{p \rightarrow 1} v(x, t)=\lim _{N \rightarrow \infty} \sum_{n=0}^{N} v_{n}(x, t) \\
& =\mathrm{e}^{-x}\left(\frac{1}{2}+\frac{t}{4}-\frac{t^{3}}{48}+\frac{t^{5}}{480}-\frac{t^{7}}{80640}+\cdots\right) \\
& =\frac{\mathrm{e}^{-x}}{1+\mathrm{e}^{-t}}
\end{aligned}
$$

which is the exact solution.

\section{Case (c) Solution by HAM}

Choosing the linear operator as $L=\frac{\partial}{\partial t}$ and using the Equations (4.8-4.10), we obtain the following $m$ th order deformation equation as

$$
\begin{aligned}
u_{m}(x, t)= & \chi_{m} u_{m-1}(x, t)+\hbar \int_{0}^{t}\left[\frac{\partial u_{m-1}(x, \tau)}{\partial \tau}+\frac{\partial u_{m-1}(x, \tau)}{\partial x}\right. \\
& \left.+\sum_{i=0}^{m-1} u_{i}(x, \tau) \int_{0}^{A} u_{m-1-i}(x, \tau) \mathrm{d} x\right] \mathrm{d} \tau, \\
& m=1,2,3, \cdots
\end{aligned}
$$

Taking the initial guess as $u_{0}(x, t)=u(x, 0)=\frac{\mathrm{e}^{-x}}{2}$, and solving the mth Equation (5.6), we get the following

$$
\begin{aligned}
& u_{1}(x, t)=-\hbar t \frac{\mathrm{e}^{-x}}{4} \\
& u_{2}(x, t)=-(1+\hbar) \hbar t \frac{\mathrm{e}^{-x}}{4} \\
& u_{3}(x, t)=-(1+\hbar)^{2} \hbar t \frac{\mathrm{e}^{-x}}{4}+\hbar^{3} t^{3} \frac{\mathrm{e}^{-x}}{48}, \cdots
\end{aligned}
$$

Truncating the series (4.7) at level $\mathrm{m}=7$, we obtain an approximate solution of (5.1) as

$$
\tilde{u}_{7}(x, t)=u_{0}(x, t)+\sum_{m=1}^{7} u_{m}(x, t),
$$

the accuracy of approximation is controlled by the auxiliary parameter $\hbar$ as illustrated by Figures 3 and 4 .

The approximate solution $\tilde{u}_{m}(x, t)$ converges to the exact solution $\frac{\mathrm{e}^{-x}}{1+\mathrm{e}^{-t}}$, as $m \rightarrow \infty$ for $\hbar=-1$.

The ADM and HPM solutions are obtained by taking $\hbar=-1$ in HAM solution.

Table 1 shows that the approximate solution $\tilde{u}_{7}(x, t)$ obtained by HAM for $\hbar=-1$ is more accurate compared to that obtain by reproducing kernel method [14].

Figure 1 shows the approximate solution for $\hbar=-1$, whereas Figures 2, 3 show the errors $E_{7}(\hbar)$ for different values of $\hbar$. It is observed that $E_{7}(-0.99)$ is smaller than $E_{7}(-1)$.

Example 5.2 Consider the following nonlinear agestructured population model $[8,13]$.

$$
\begin{gathered}
\frac{\partial u(x, t)}{\partial t}+\frac{\partial u(x, t)}{\partial x}=-(P(t)+1) u(x, t), \\
t \geq 0,0 \leq x<A,
\end{gathered}
$$

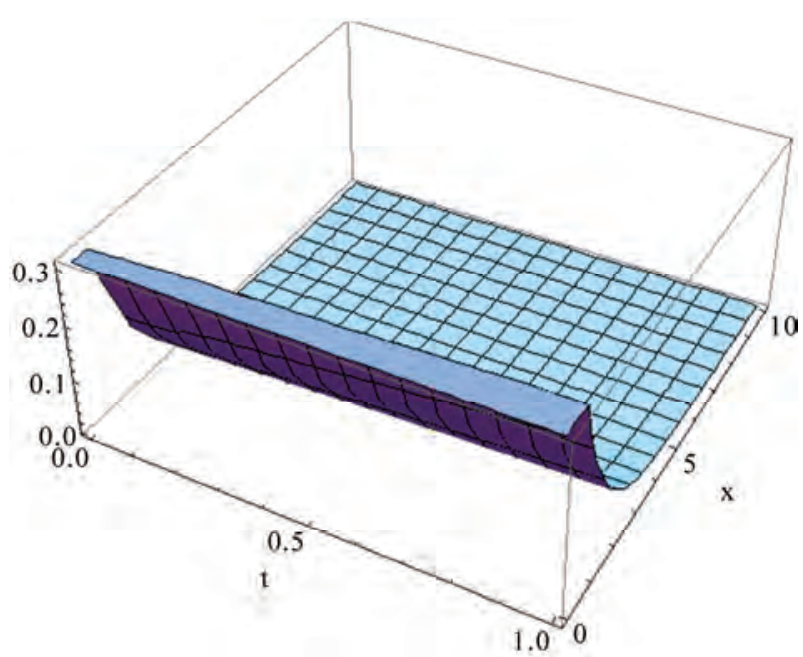

Figure 1. Approximate solution $\tilde{u}_{7}(x, t), \quad \hbar=-1$.

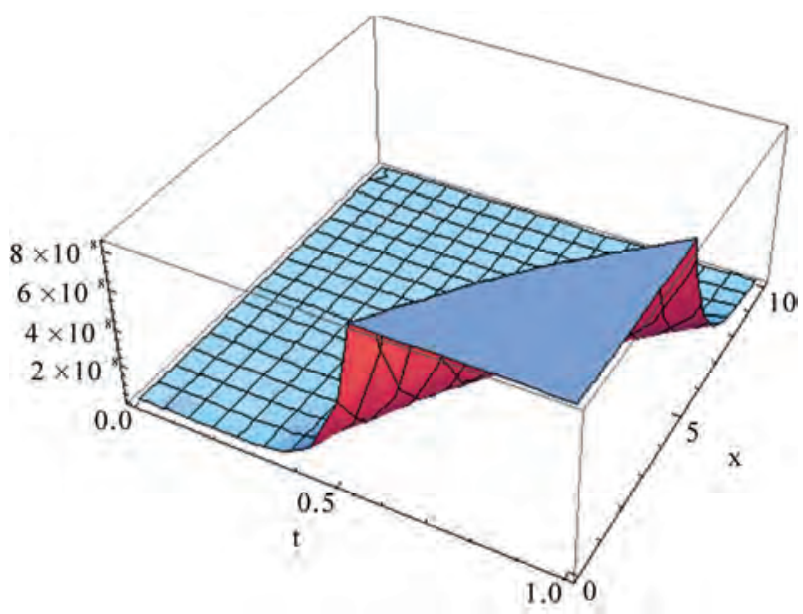

Figure 2. Error $E_{7}(-1)$. 


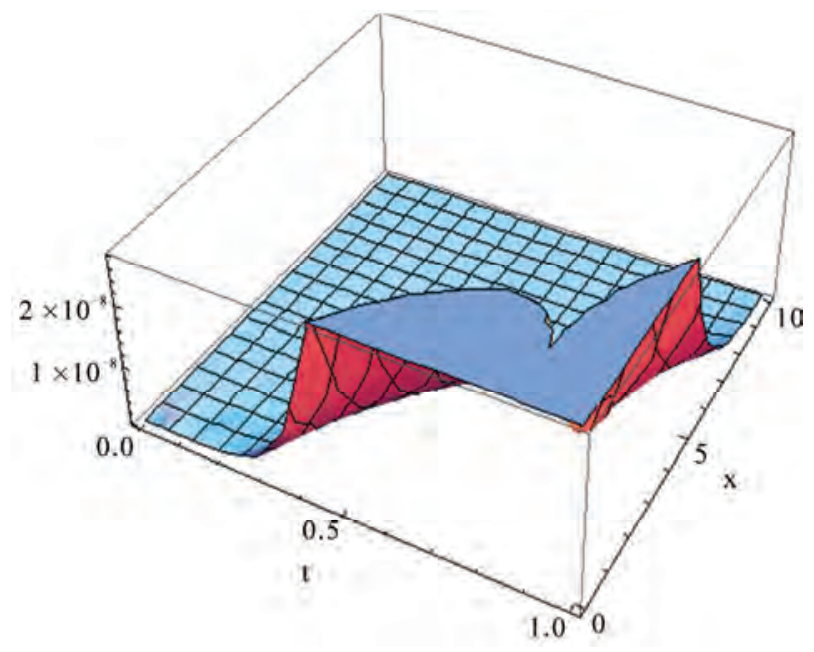

Figure 3. Error $E_{7}(-0.99)$.

$$
\begin{gathered}
u(x, 0)=\frac{\mathrm{e}^{-x}}{2}, 0 \leq x<A, \\
u(0, t)=P(t), t \geq 0, \\
P(t)=\int_{0}^{A} u(x, t) \mathrm{d} x, t \geq 0,
\end{gathered}
$$

where $A=+\infty$. It is easy to verify that $u(x, t)=\frac{\mathrm{e}^{-x}}{2+t}, t \geq 0, x \geq 0$, is the exact solution of (5.7).

\section{Case (a) Solution by ADM}

Rewriting Equation (5.7) in operator form

$$
L_{t} u(x, t)=-L_{x} u(x, t)-u(x, t)-u(x, t) \int_{0}^{A} u(x, t) \mathrm{d} x,
$$

taking initial approximation $u_{0}(x, t)=\frac{e^{-x}}{2}$, and using the recursive formula (2.7), we get

$$
\begin{aligned}
& u_{1}(x, t)=-t \frac{\mathrm{e}^{-x}}{4}, u_{2}(x, t)=t^{2} \frac{\mathrm{e}^{-x}}{8}, u_{3}(x, t)=-t^{3} \frac{\mathrm{e}^{-x}}{16}, \\
& u_{4}(x, t)=t^{4} \frac{\mathrm{e}^{-x}}{32}, u_{5}(x, t)=-t^{5} \frac{\mathrm{e}^{-x}}{64}, \cdots
\end{aligned}
$$

The solution is given by

$$
\begin{aligned}
u(x, t) & =\lim _{N \rightarrow \infty} \sum_{n=0}^{N} u_{n}(x, t) \\
& =\mathrm{e}^{-x}\left(\frac{1}{2}-\frac{t}{4}+\frac{t^{2}}{8}-\frac{t^{3}}{16}+\frac{t^{4}}{32}-\frac{t^{5}}{64}+\frac{t^{6}}{128}-\frac{t^{7}}{256}+\cdots\right) \\
& =\frac{\mathrm{e}^{-x}}{2+t},
\end{aligned}
$$

which is the exact solution.

\section{Case (b) Solution by HPM}

Taking initial approximation $u_{0}(x, t)=\frac{\mathrm{e}^{-x}}{2}$, and using the Equations (3.10), we get

$$
\begin{aligned}
& v_{1}(x, t)=-t \frac{\mathrm{e}^{-x}}{4}, v_{2}(x, t)=t^{2} \frac{\mathrm{e}^{-x}}{8}, v_{3}(x, t)=-t^{3} \frac{\mathrm{e}^{-x}}{16}, \\
& v_{4}(x, t)=t^{4} \frac{\mathrm{e}^{-x}}{32}, v_{5}(x, t)=-t^{5} \frac{\mathrm{e}^{-x}}{64}, \cdots .
\end{aligned}
$$

Substituting these values in Equation (3.11), the solution is given by

$$
\begin{aligned}
u(x, t) & =\lim _{p \rightarrow 1} v(x, t)=\lim _{N \rightarrow \infty} \sum_{n=0}^{N} v_{n}(x, t) \\
& =\mathrm{e}^{-x}\left(\frac{1}{2}-\frac{t}{4}+\frac{t^{2}}{8}-\frac{t^{3}}{16}+\frac{t^{4}}{32}-\frac{t^{5}}{64}+\frac{t^{6}}{128}-\frac{t^{7}}{256}+\cdots\right) \\
& =\frac{\mathrm{e}^{-x}}{2+t},
\end{aligned}
$$

which is the exact solution.

\section{Case (c) Solution by HAM}

Choosing the linear operator as $L=\frac{\partial}{\partial t}$ and using the Equations (4.8-4.10), we obtain the following $m t h$ order deformation equation as

$$
\begin{aligned}
u_{m}(x, t)= & \chi_{m} u_{m-1}(x, t)+\hbar \int_{0}^{t}\left[\frac{\partial u_{m-1}(x, \tau)}{\partial \tau}+\frac{\partial u_{m-1}(x, \tau)}{\partial x}\right. \\
& \left.+u_{m-1}(x, \tau)+\sum_{i=0}^{m-1} u_{i}(x, \tau) \int_{0}^{A} u_{m-1-i}(x, \tau) \mathrm{d} x\right] \mathrm{d} \tau, \\
& m=1,2,3, \cdots
\end{aligned}
$$

Taking the initial guess as $u_{0}(x, t)=u(x, 0)=\frac{e^{-x}}{2}$, and solving the mth Equation (5.12), we get the following

$$
\begin{aligned}
& u_{1}(x, t)=\hbar t \frac{\mathrm{e}^{-x}}{4}, \\
& u_{2}(x, t)=(1+\hbar) \hbar t \frac{\mathrm{e}^{-x}}{4}+\hbar^{2} t^{2} \frac{\mathrm{e}^{-x}}{8}, \\
& u_{3}(x, t)=(1+\hbar)^{2} \hbar t \frac{\mathrm{e}^{-x}}{4}+2(1+\hbar) \hbar^{2} t^{2} \frac{\mathrm{e}^{-x}}{8}+\hbar^{3} t^{3} \frac{\mathrm{e}^{-x}}{16}, \cdots
\end{aligned}
$$

Truncating the series (4.7) at level $m=8$, we obtain an approximate solution of (5.7) as

$$
\tilde{u}_{8}(x, t)=u_{0}(x, t)+\sum_{m=1}^{8} u_{m}(x, t) .
$$


The approximate solution $\tilde{u}_{m}(x, t)$ converges to the exact solution $\frac{\mathrm{e}^{-x}}{2+t}$, as $m \rightarrow \infty$ for $\hbar=-1$.

Figure 4 shows the approximate solution for $\hbar=-1$, whereas Figures 5, 6 show the errors $E_{8}(\hbar)$ for different values of $\hbar$. It is observed that $E_{8}(-0.7905)$ is smaller than $E_{8}(-1)$.

In the next example, we consider a SIS model describing the evolution of a human nonlethal disease which does not import immunity [23-25]. Some infections, for example the group of those responsible for the common cold, do not confer any long lasting immunity. Such infections do not have a recovered state and individuals become susceptible again after infection. Maybe, the most specific parameter of biological system is the age, and, especially for some infectious diseases, it has a deep influence on the dynamics of its spreading in a population. Many of the parameters may depend on age, especially the contact rate, which summarizes the 'infectious effectiveness' of contacts between susceptible and infectious subjects. This effectiveness has, thus, to take into account both the age of the infectious and the age of the susceptible. Epidemic models modelling the age structure of a population are very complex. Example (5.3) illustrates the utility of our algorithm on such types of complex models.

Example 5.3 We consider the following age-structured SIS model [13]:

$$
\begin{gathered}
\frac{\partial u(x, t)}{\partial t}+\frac{\partial u(x, t)}{\partial x}+\frac{1}{1-x} u(x, t)=(i(x, t)-u(x, t)) P(t), \\
t \geq 0,0 \leq x<A \\
u(x, 0)=2(1-x), \quad 0 \leq x<A \\
u(0, t)=\int_{0}^{A} \beta(x, i) u(x, t) \mathrm{d} x, t \geq 0 \\
P(t)=\int_{0}^{A} u(x, t) \mathrm{d} x, t \geq 0
\end{gathered}
$$

where $A=1, \beta=4(1-x)^{2}$ or $\beta=\pi \sin \pi x$.

For the total population, we take a steady state distribution such that $i(x, t)=4(1-x)$. Then the exact solution of (5.13) is $u(x, t)=\frac{4(1-x)}{1+e^{-2 t}}$.

Following the procedures adopted in the previous examples, we apply the three methods and obtain the various iterates of the solution as follows:

As ADM and HPM iterates are identical for $p=1$, we list them once.

1) ADM and HPM iterates

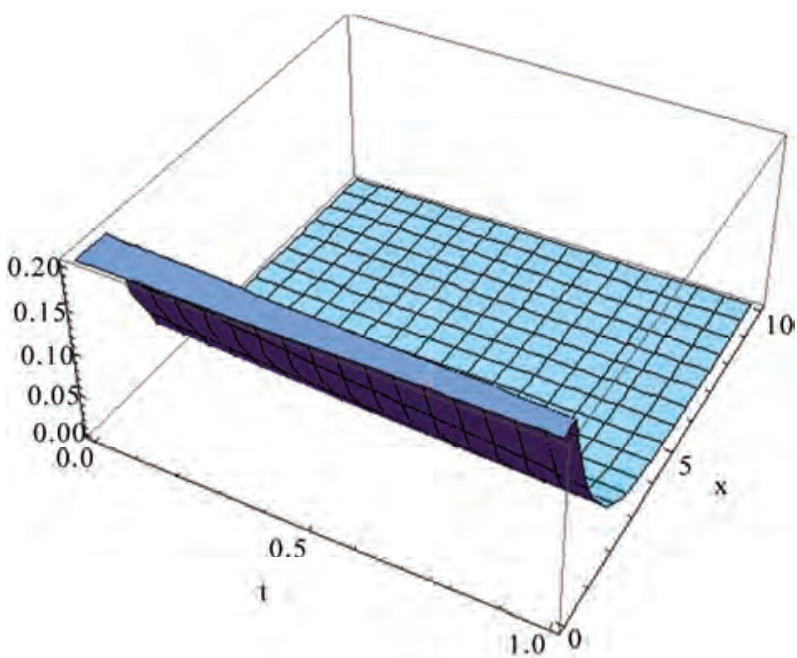

Figure 4. Approximate solution $\tilde{u}_{8}(x, t), \quad \hbar=-1$.

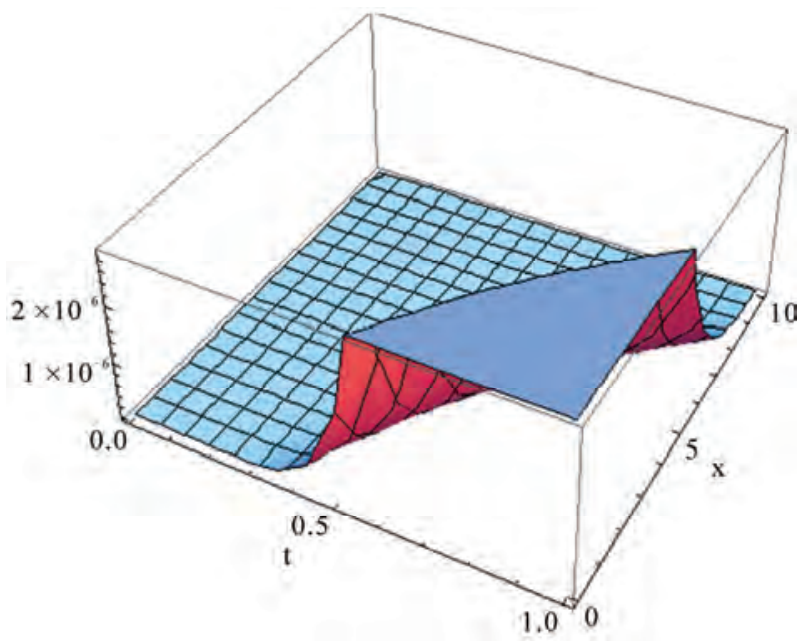

Figure 5. Error $E_{8}(-1)$.

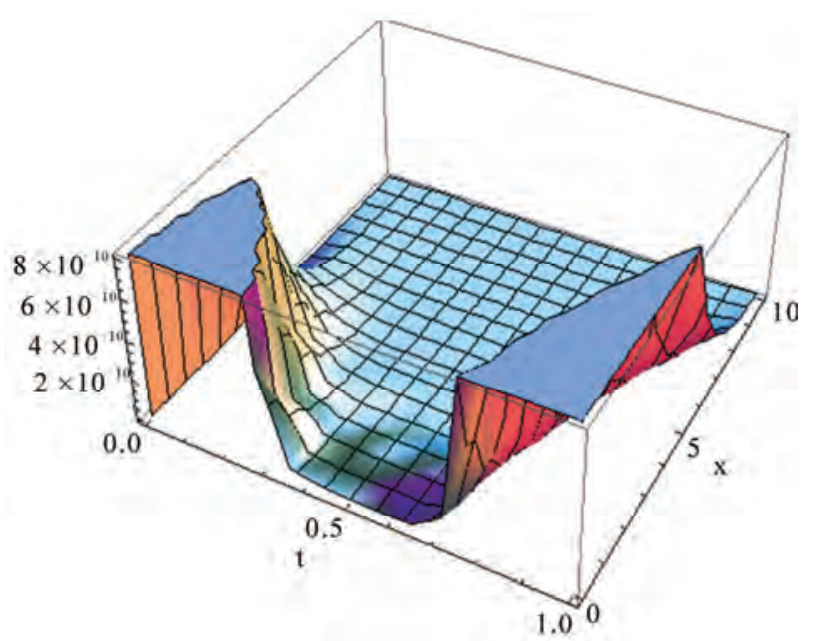

Figure 6. Error $E_{8}(-0.7905)$. 


$$
\begin{gathered}
u_{0}(x, t)=2(1-x), u_{1}(x, t)=2 t(1-x), \\
u_{3}(x, t)=-\frac{2}{3} t^{3}(1-x), u_{5}(x, t)=\frac{4}{15} t^{5}(1-x), \\
u_{7}(x, t)=-\frac{34}{315} t^{7}(1-x), u_{9}(x, t)=\frac{124}{2835} t^{9}(1-x), \\
u_{11}(x, t)=-\frac{3268}{155925} t^{11}(1-x), u_{2 n}(x, t)=0, n=1,2, \cdots .
\end{gathered}
$$

The solution is given by

$$
\begin{aligned}
u(x, t) & =\lim _{N \rightarrow \infty} \sum_{n=0}^{N} u_{n}(x, t) \\
& =2(1-x)\left(1+t-\frac{t^{3}}{3}+\frac{2 t^{5}}{15}-\frac{17 t^{7}}{315}+\frac{62 t^{9}}{2835}-\frac{1634 t^{11}}{155925}+\cdots\right) \\
& =\frac{4(1-x)}{1+\mathrm{e}^{-2 t}}
\end{aligned}
$$

which is the exact solution.

\section{2) HAM iterates}

$u_{0}(x, t)=2(1-x)$,

$u_{1}(x, t)=-2 \hbar t(1-x)$,

$u_{2}(x, t)=-2(1+\hbar) \hbar t(1-x)$,

$u_{3}(x, t)=-2(1+\hbar)^{2} \hbar t(1-x)+\frac{2}{3} \hbar^{3} t^{3}(1-x)$,

$u_{4}(x, t)=-2(1+\hbar)^{3} \hbar t(1-x)+2(1+\hbar) \hbar^{3} t^{3}(1-x), \cdots$.

Truncating the series (4.7) at the level $m=11$, we obtain an approximate solution of (5.13) as

$$
\tilde{u}_{11}(x, t)=u_{0}(x, t)+\sum_{m=1}^{11} u_{m}(x, t) .
$$

The approximate solution $\tilde{u}_{m}(x, t)$ converges to the exact solution $\frac{4(1-x)}{1+\mathrm{e}^{-2 t}}$, as $m \rightarrow \infty$ for $\hbar=-1$.

Figure 7 shows the approximate solution for $\hbar=-1$, whereas Figures 8, 9 show the errors $E_{11}(\hbar)$ for different values of $\hbar$. It is observed that $E_{11}(-0.95312)$ is smaller than $E_{11}(-1)$.

\section{Conclusion}

In this paper, we have given three simple, easy to implement analytic algorithms based on ADM, HPM and HAM for nonlinear models of age-structured population dynamics and epidemiology. These algorithms involve infinite convergent series and in many cases, the closed form solutions are obtained. Where the closed form analytical solutions are not readily available, it is established through the three numerical experiments that truncating the solution series at relatively lower level of truncation give fairly accurate solutions. Moreover, the accuracy of

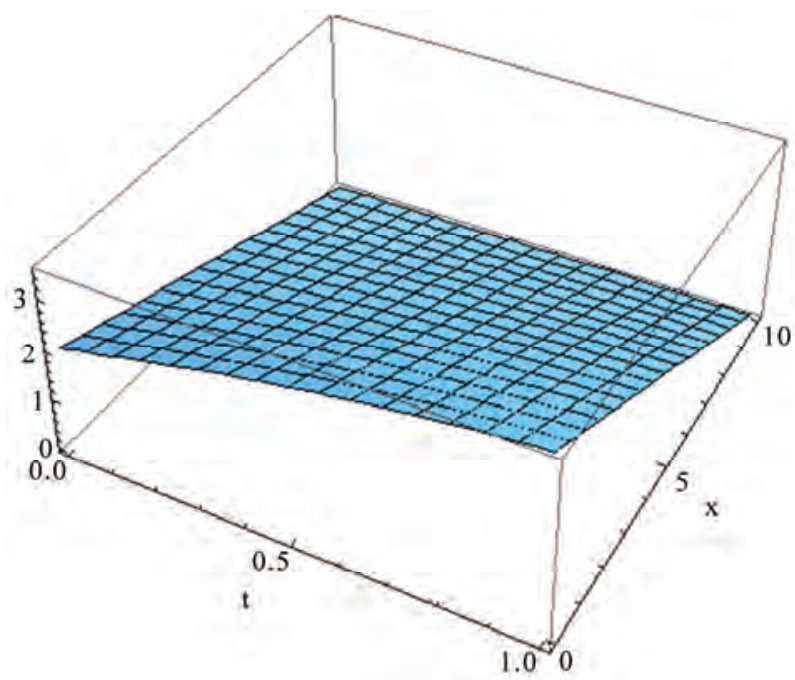

Figure 7. Approximate solution $\tilde{u}_{11}(x, t), \quad \hbar=-1$.

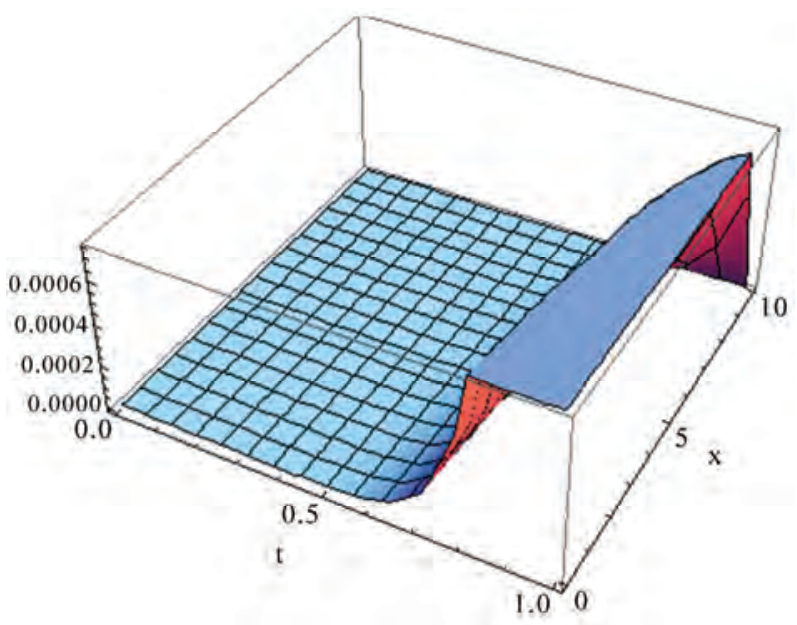

Figure 8. Error $E_{11}(-1)$.

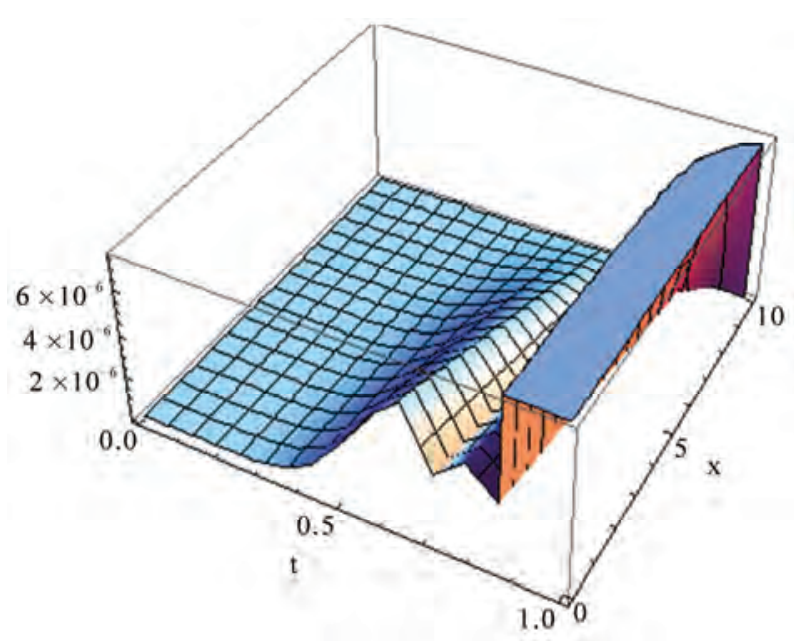

Figure 9. Error $E_{11}(-0.95312)$. 
the solution may be increased by the suitable choice of the auxiliary parameter $\hbar$ in HAM. Table 1 clearly establishes the accuracy of our method compared to that of the reproducing kernel method proposed by Cui and Chen [14].

\section{Acknowledgements}

The first and third authors acknowledge the financial support from UGC and CSIR New-Delhi, India, respectively under JRF schemes.

\section{References}

[1] L. Sigler, F. L. Abaci, “A Translation into Modern English of Leonardo Pisano's Book of Calculation,” Springer-Verlag, New-York, 2002.

[2] T. R. Malthus, "An Essay on the Principle of Population, St. Paul's, London,” 1798, In: T. R. Malthus, “An Essay on the Principle of Population and A Summary View of the Principle of Population,” Penguin, Harmondsworth, England, 1970.

[3] F. R. Sharpe and A. J. Lotka, "A Problem in Age Distributions,” Philosophical Magzine, Vol. 21, No. 124, 1911, pp. 435-438.

[4] A. G. McKendrick, "Applications of Mathematics to Medical Problems,” Proceedings of Edinburgh Mathematical Society, Vol. 44, 1926, pp. 98-130. doi:10.1017/S0013091500034428

[5] M. E. Gurtin and R. C. MacCamy, "Nonlinear Age-Dependent Population Dynamics," Archive for Rational Mechanics and Analysis, Vol. 54, No. 3, 1974, pp. 281300.

[6] M. Iannelli, "Mathematical Theory of Age-Structured Population Dynamics," Applied Mathematics Monographs, Vol. 7, Consiglio Nazionale delle Ricerche, Pisa, 1995.

[7] G. F. Webb, “Theory of Nonlinear Age-Dependent Population Dynamics,” Marcel Dekker, New York, January 1985.

[8] X. Y. Li, "Variational Iteration Method for Nonlinear Age-Structured Population Models," Computers and Mathematics with Applications, Vol. 58, No. 11-12, 2009, pp. 2177-2181. doi:10.1016/j.camwa.2009.03.060

[9] L. M. Abia and J. C. Lopez-Marcos, "Runge-Kutta Methods for Age-Structured Population Models,” Applied Numerical Mathematics, Vol. 17, No. 1, 1995, pp. 1-17. doi:10.1016/0168-9274(95)00010-R

[10] L. M. Abia and J. C. Lopez-Marcos, "On the Numerical Integration of Non-Local Terms for Age-Structured Population Model,” Mathematical Biosciences, Vol. 157, No.1, 1999, pp. 147-167. doi:10.1016/S0025-5564(98)10080-9

[11] L. M. Abia, O. Angulo and J. C. Lopez-Marcos, “AgeStructured Population Models and Their Numerical Solution,” Ecological Modelling, Vol. 188, No. 1, 2005, pp. 112-136. doi:10.1016/j.ecolmodel.2005.05.007
[12] M. Y. Kim and E. J. Park, "An Upwind Scheme for a Nonlinear Model in Age-Structured Population Dynamics," Computers and Mathematics with Applications, Vol. 30, No. 8, 1995, pp. 5-17. doi:10.1016/0898-1221(95)00132-I

[13] M. Iannelli, M. Y. Kim and E. J. Park, "Splitting Method for the Numerical Approximation of Some Models of Age-Structured Population Dynamics and Epidemiology,” Applied Mathematics and Computation, Vol. 87, No. 1, 1997, pp. 69-93. doi:10.1016/S0096-3003(96)00222-6

[14] M. G. Cui and C. Chen, "The Exact Solution of Nonlinear Age-Structured Population Model,” Nonlinear Analysis: Real World Applications, Vol. 8, No. 4, 2007, pp. 1096-1112. doi:10.1016/j.nonrwa.2006.06.004

[15] P. Krzyzanowski, D. Wrzosek and D. Wit, "Discontinuous Galerkian Method for Piecewise Regular Solution to the Nonlinear Age-Structured Population Model," Mathematical Biosciences, Vol. 203, No. 2, 2006, pp. 277-300. doi:10.1016/j.mbs.2006.05.005

[16] Norhayati and G. C. Wake, "The Solution and the Stability of a Nonlinear Age-Structured Population Model," Journal of the Australian Mathematical Society, Vol. 45, 2003, pp. 153-165. doi:10.1017/S1446181100013237

[17] G. Adomian, "A Review of the Decomposition Method in Applied Mathematics,” Journal of Mathematical Analysis and Applications," Vol. 135, No. 2, 1988, pp. 501-544. doi:10.1016/0022-247X(88)90170-9

[18] G. Adomian, "Solving Frontier Problems of Physics: The Decomposition Method,” Kluwer Academic Publishers, Boston, 1999.

[19] J. H. He, “Homotopy Perturbation Technique,” Computer Methods in Applied Mechanics and Engineering, Vol. 178, No. 3, 1999, pp. 257-262. doi:10.1016/S0045-7825(99)00018-3

[20] S. J. Liao, "Beyond Perturbation: Introduction to Homotopy Analysis Method,” Chapman \& Hall/CRC Press, Bosca Raton, December 2003.

[21] M. Dehghan and R. Salehi, "Solution of a Nonlinear Time-Delay Model in Biology via Semi-Analytical Approaches,” Computer Physics Communication, Vol. 181, No. 7, 2010, pp. 1255-1265. doi:10.1016/j.cpc.2010.03.014

[22] S. J. Liao and Y. Tan, "A General Approach to Obtain Series Solutions of Nonlinear Differential Equations," Studies in Applied Mathematics, Vol. 119, No. 4, 2007, pp. 297-355. doi:10.1111/j.1467-9590.2007.00387.x

[23] S. Busenberg, K. Cooke and M. Iannelli, "Endemic Thresholds and Stability in a Class of Age-Structured Epidemics,” SIAM Journal Applied Mathematics, Vol. 48, No. 6, December 1988, pp. 1379-1395. doi: $10.1137 / 0148085$

[24] S. Busenberg, M. Iannelli and H. Thieme, "Global Behaviour of an Age Structured Epidemic Model," SIAM Journal on Mathematical Analysis, Vol. 22, No. 4, July 1991, pp. 1065-1080. doi:10.1137/0522069 
[25] M. Iannelli, F. Milner and A. Pugliese, “Analytical and Numerical Results for the Age Structured SIS Epidemic Model with Mixed Inter-Intracohort Transmission,”
SIAM Journal on Mathematical Analysis, Vol. 23, No. 3, May 1992, pp. 662-688. doi:10.1137/0523034 